\title{
Twisting of the Ranger Drug-Coated Balloon for Treating Superficial Femoral Artery Disease
}

\author{
Su Hong Kim ${ }^{1}$, Jong Hyun $\mathrm{Choi}^{1}$, Bo Won Kim ${ }^{1}$, and Sang Hee Kim² \\ ${ }^{1}$ Busan Veterans Hospital \\ ${ }^{2}$ Inje University - Busan Campus
}

November 18, 2021

\begin{abstract}
Paclitaxel-coated balloons have demonstrated improved efficacy compared with uncoated percutaneous transluminal angioplasty for femoropopliteal artery disease. While applying a long balloon, twisting of the balloon can be occurred. We reported two cases with twisting of the Ranger drug-coated balloon for treating superficial femoral artery.

Twisting of the Ranger Drug-Coated Balloon for Treating Superficial Femoral Artery Disease

Su Hong Kim ${ }^{1}$, Jong Hyun $\mathrm{Choi}^{1}$, Bo Won Kim ${ }^{1}$, Sang Hee Kim ${ }^{2}$

${ }^{1}$ Department of Cardiovascular Medicine, Busan Veterans Hospital, Korea Veterans Health Service, Busan, Republic of Korea ${ }^{2}$ College of Nursing, Inje University, Busan, Republic of Korea

Correspondance

Su Hong Kim, Department of Cardiovascular Medicine, Busan Veterans Hospital, Korea Veterans Health Service, 420 Baekyangdae-ro, Sasang-gu, Busan 46996, Republic of Korea

Email:dhugie@naver.com

Abstract

Paclitaxel-coated balloons have demonstrated improved efficacy compared with uncoated percutaneous transluminal angioplasty for femoropopliteal artery disease. While applying a long balloon, twisting of the balloon can be occurred. We reported two cases with twisting of the Ranger drug-coated balloon for treating superficial femoral artery.
\end{abstract}

\section{KEYWORDS}

Peripheral Arterial Disease; Femoral Artery; Angioplasty; Torsion, Mechanical

\section{INTRODUCTION}

With the aging of the population, the incidence of peripheral artery disease (PAD) is increasing, and intermittent claudication (IC) is the most common symptom of peripheral artery disease, and it is known that more than $20 \%$ of the aged 70 years or older experience it. ${ }^{1)}$

Superficial femoral artery (SFA) is one of the longest and most dynamically active vessels in the body undergoing torsion, compression, flexion and extension from leg motion, and is known to be more susceptible to atherosclerosis. ${ }^{2)}$ An Endovascular therapy (EVT)-first strategy is usually recommended in femoropopliteal (FP) artery disease. Even with high technical success rate, plain old balloon angioplasty (POBA) is burdened with high restenosis rate. To reduce this problem, local delivery of drugs has been proposed by way 
of drug-coated balloons (DCB). DCB have demonstrated improved efficacy compared with uncoated percutaneous transluminal angioplasty (PTA) for FP artery. ${ }^{3)}$ The use of self-expanding nitinol stents (SES) had the advantage of a higher rate patency rate than POBA, but the occurrence of in-stent restenosis (ISR) has become a problem, occurring in up to $40 \%$ of patients within the first year. ${ }^{4) 5)}$ A recent meta-analysis comparing DCB versus POBA for FP ISR showed that the incidence of combined recurrent ISR in the DCB group was significantly lower than that in the POBA group, consequently freedom from TLR in the DCB group was significantly higher than that in the POBA group. ${ }^{6}$ ) The superior efficacy results of DCB over POBA have made DCB an important method for the treatment of FP ISR.

All current available DCBs use paclitaxel, but have difference in drug concentration, excipient, elution kinetics and profile. The Ranger Paclitaxel-Coated PTA Balloon Catheter (Boston Scientific, Marlborough, Massachusetts) is a commonly used DCB for the treatment of FP artery disease because it has a lower paclitaxel dose and longer balloon profile than other DCBs.

During applying a long balloon in a long and complex lesion with aggressive manipulation, the balloon can twist inside the lesions, only one case report of twisting of the peripheral balloon used in the peroneal artery has been reported in the literature. ${ }^{7)}$ Twisting of the DCB for FP artery disease has not been reported in the literature.

Here we reported two cases of twisting of the Ranger DCB for treating FP artery disease that was solved through collaboration with a manufacturing company.

\section{CASE PRESENTATION}

\section{[CASE 1]}

A 73 year old man with history of hypertension, dyslipidemia presented to our hospital with complaints of IC for 3 months. The intermittent claudication had gradually aggravated, accompanied by the resting pain for a month. His past medical history included cholecystectomy for acute cholecystitis and EVT for PAD. A year ago, a SES was deployed at the distal SFA and a drug eluting stent was deployed at the proximal SFA for treating complete occlusion of the SFA. His ankle brachial pressure index (ABI) was 0.50 in the right leg and 1.00 in the left leg, and both femoral arteries were palpable. His heart and lung functions were normal. Blood sampling test showed a creatinine level of $1.3 \mathrm{mg} / \mathrm{dL}$. He was ex-smoker with 10 years history of smoking and did not have diabetes. Subsequently, patient underwent peripheral angiogram, and this showed total occlusion from proximal to distal SFA with ISR, the blood flow was restored via collaterals from the deep femoral artery to the distal SFA (Figure 1A). We planned endovascular therapy for the SFA. Initially wire crossing was done with Gladius, Halberd and Astato XS wire and CXI supporting catheter. For the predilation, a $5.0 \times 200 \mathrm{~mm}$ Admiral Xtreme PTA balloon catheter (Medtronic, Roncadelle(BS), Italy) was advanced and crossed the lesion easily. We inflated the balloon 2 times from the distal SFA to common femoral artery (CFA). To obtain better clinical results after predilation, we decided to use DCB. After applying a $5.0 \times 200 \mathrm{~mm}$ Ranger DCB from the middle to the distal SFA, a Ranger DCB of the same size was applied from the middle SFA to the ostium of SFA. During the inflation of the second Ranger DCB at 10 ATM (norminal pressure 6, rated burst pressure 14 ATM), we noticed a significant wasting in inflation in the middle short segment of DCB (Figure 1B). The defect was not detected during predilation in a same location suggestive of problem within the DCB rather than a non-dilatable lesion. Final angiogram after intervention revealed less than $30 \%$ stenosis with good flow. Clinical improvement was seen in 1 months follow up with improvement of walking distance without claudication.

\section{[CASE 2]}

A 73 year old male Vietnam war veterans who exposed to agent orange with history of hypertension, dyslipidemia, chronic kidney disease presented with complaints of IC for 8 months. The intermittent claudication had gradually aggravated for 8 months. He could not walk because he had claudication even after walking 100 meters and the claudication of the left leg was more severe than that of the right. His past medical history included spinal stenosis, benign prostate hyperplasia, nephrolithiasis and cholecystectomy for cholelithiasis. 
His ABI was 0.83 in the right leg and 0.85 in the left leg. Although the claudication was severe, the ABI was not as bad as expected. His heart and lung functions were normal. Blood sampling test showed a creatinine level of $1.1 \mathrm{mg} / \mathrm{dL}$. He was current smoker with 50-years history of smoking and did not have diabetes. He underwent the lower extremity computed tomography (CT) angiography and this showed multiple stenosis from the proximal SFA to the mid SFA and stenosis at the tibioperoneal trunk (TPT). Subsequently, he underwent peripheral angiogram, and findings were almost same to those of CT (Figure 2A). We planned endovascular therapy for the SFA and TPT. Initially wire crossing was done with Gladius and CXI supporting catheter. For the predilation, a 5.0x 80mm Powerflex Pro PTA dilatation catheter (Cordis, Alajuela, Costa Rica) was advanced and crossed the lesion easily. We inflated the balloon 4 times from the distal SFA to the proximal SFA. The lesion at the TPT was dilated with a 4x 120mm Armada 18 PTA catheter (Abbott Vascular, Alajuela, Costa Rica). To obtain better clinical results after predilation, we decided to use DCB for treating SFA, a $5.0 \mathrm{x} 200 \mathrm{~mm}$ Ranger DCB was applied from the mid SFA to the proximal SFA. During the inflation of the Ranger DCB at 8 ATM, we noticed a significant wasting in inflation in the middle short segment of DCB (Figure 2B). The wasting remained despite raising pressure to the rated burst pressure. Final angiogram after intervention revealed good flow. Clinical improvement was seen in 1 months follow up with improvement of walking distance without claudication.

After the intervention the balloon was sent to Boston Scientific for further analysis which revealed small middle part of balloon does not fully inflate due to the presence a twist in the balloon. Manufacturing company's investigation revealed that there were possible problems with the carrier hoop for the curvature set or balloon rotation while loading the tool over the balloon. Manufacturing company has redesigned the packing of the balloon in order to reduce the occurrence of a balloon twist. First, they reduced carrier hoop diameter with additional coiling plate and second, extended the length of the carrier tube to account for catheter shifting.

\section{Discussion}

For revascularization of the FP artery, EVT plays a primary role and DCB are widely used with better clinical results than $\mathrm{POBA}^{3)}$. Ranger DCB demonstrated consistent results with nearly $90 \%$ patency at 12-months in the RANGERT II SFA and COMPARE Trials and is built on the 0.018 inch balloon platform with 0.018 and 0.014 inch guidewire compatibility. ${ }^{8)}$ Twisting of peripheral balloon during below the knee artery intervention has been reported but twisting of DCB for treating FP artery disease has not been described before. ${ }^{7}$ ) This may be caused by several reasons. First, difficulty in advancing the balloon with aggressive manipulation through the tortuous iliac artery to the femoropopliteal artery lesion might have caused. Second, it might have occurred due to the manufacturing problems mentioned previously and third, a longer length profile of the Ranger DCB compared to other DCBs might cause twisting. After identifying and improving possible causes with quick cooperation between the manufacturing company and the clinician, this problem no longer occurred.

Conclusion

We have reported an unexpected twisting of the Ranger DCB for treating FP artery disease. If focal underexpansion of a long peripheral balloon occurred, twisting of balloon should be considered and close cooperation with the manufacturer is important to solve the problem.

\section{ACKNOWLEDGEMENTS}

We would like to thank Boston Scientific for showing quick response when problem occurred and for helping us report these important cases.

\section{CONFLICT OF INTEREST}

The authors have no conflict of interest to declare.

\section{AUTHOR CONTRIBUTIONS}


Su Hong Kim : corresponding author, contributed to the writing, editing, clinical follow up of patients and cooperation with the manufacturing company.

Jong Hyun Choi : contributed to the writing and editing and cooperation with the manufacturing company.

Bo Won Kim : contributed to the writing and editing and cooperation with the manufacturing company.

Sang Hee Kim : contributed to the writing and editing.

\section{ETHICAL APPROVAL}

Writtent informed consents were obtained from patients to use clinical information and medical images, and publish this report in accordance with journal's patent consent policy.

\section{REFERENCES}

1. Norgren L, Hiatt WR, Dormandy JA, Nehler MR, Harris KA, Fowkes FG. Inter-Society Consensus for the Management of Peripheral Arterial Disease (TASC II). J Vasc Surg 2007;45:S5-67

2. Otsuka F, Nakano M, Sakakura K, Ladich E, Kolodgie FD, Virmani R, Unique demandes of the femoral artery anatomy and pathology and the need for unique interventions. J Cardiovasc Surg (Torino). 2013;54(2):191-210

3. Caradu C, Lakhlifi E, Colacchio EC, Midy D, Berard X, Poirier M, Ducasse E. Systematic review and updated meta-analysis of the use of drug-coated balloon angioplasty versus plain old balloon angioplasty for femoropopliteal arterial disease. J Vasc Surg 2019;70-981-95.e10

4. Schillinger M, Sabeti S, Loewe C, et al. Balloon angioplasty versus implantation of nitinol stents in the superficial femoral artery. N Engl J Med. 2006;354:1879-88.

5. Soga Y, Iida O, Hirano K, et al. Mid-term clinical outcome and predictors of vessel patency after femoropopliteal stenting with self-expandable nitinol stent. J Vasc Surg. 2010;52:608-15.

6. Wu R, Zilun L, Wang M, et al. Paclitaxel-coated versus uncoated balloon angioplasty for femoropopliteal artery in-stent restenosis. Int J Surg. 2017;42:72-82.

7. Akkus NI, Bahadur F, Varma J. Twisting of peripheral balloon appearing as a non-dilating peroneal artery lesion. Cardiovasc Revasc Med . 2013;14:41-4

8. Sachar R, Soga Y, Ansari MM, et al. 1-Year Results From the RANGER II SFA Randomized Trial of the Ranger Drug-Coated Balloon. JACC Cardiovasc Interv . 2021;14:1123-33

Figure 1. A. Angiogram showed total occlusion from proximal to distal superficial femoral artery with instent restenosis, the blood flow was restored via collaterals from the deep femoral artery to the distal superficial femoral artery. B. Angiogram showed a significant wasting in inflation in the middle short segment the Ranger Drug-Coated Balloon.

Figure 2. A. Angiogram showed multiple stenosis from the proximal superficial femoral artery to the mid superficial femoral artery. B. Angiogram showed a significant wasting in inflation in the middle short segment the Ranger Drug-Coated Balloon. 


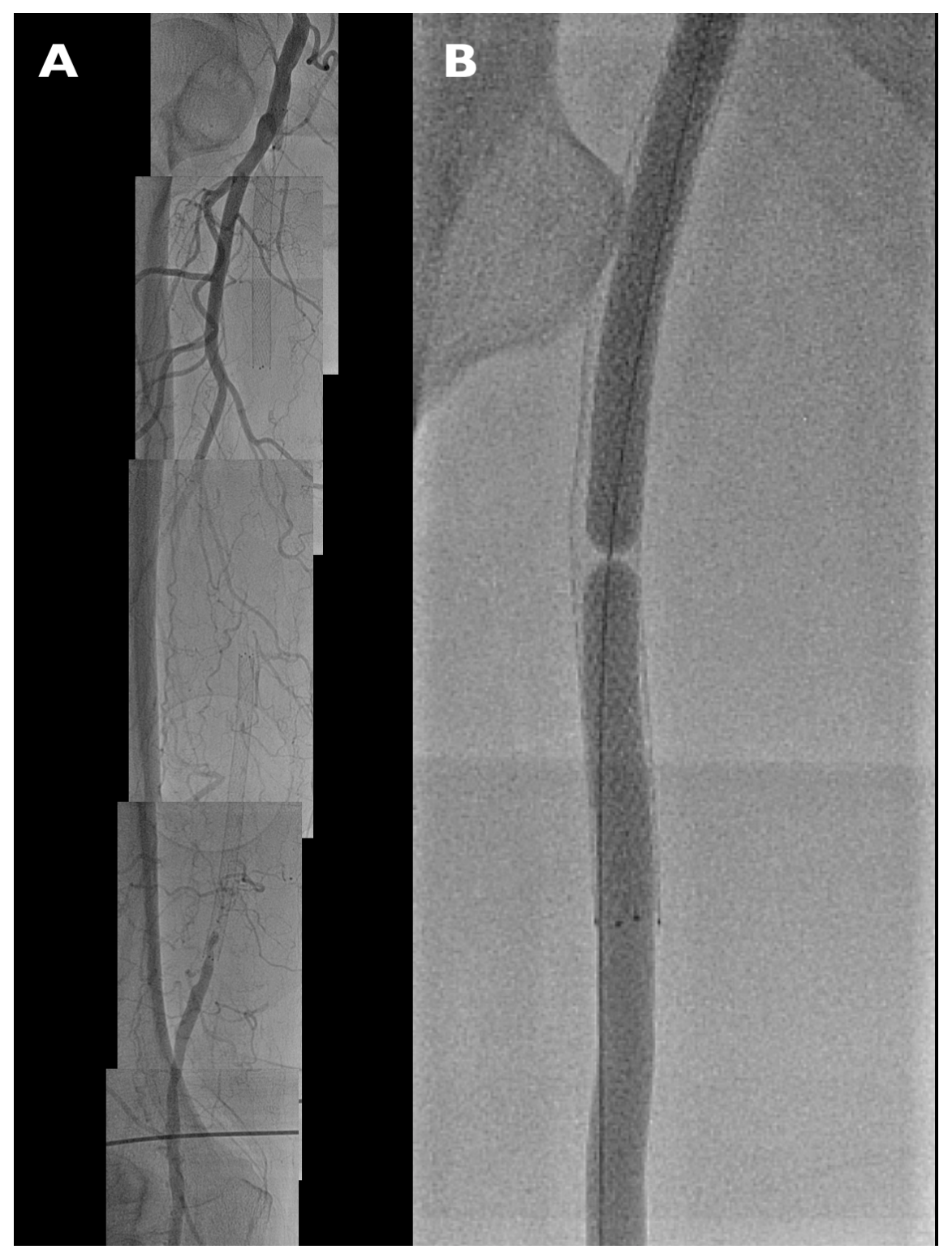




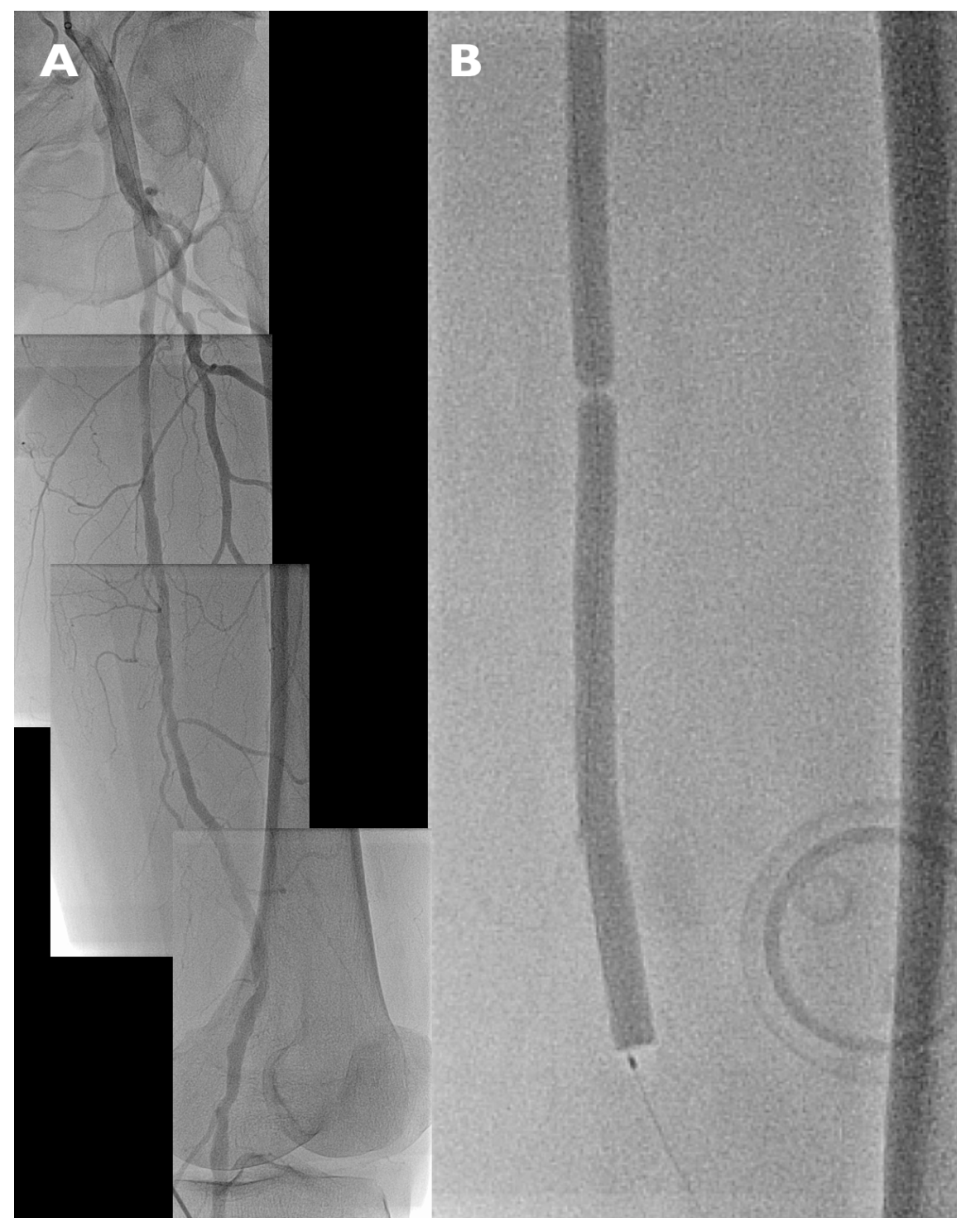

